\title{
A Unified CPE for Multiple Mainstream IPv4/IPv6 Transition Mechanisms
}

\author{
Shuo Liu, ${ }^{1, a}$, Guoliang Han ${ }^{1, b,}$ Congxiao Bao ${ }^{1, c}$, Xing Li ${ }^{1, d}$ \\ ${ }^{1}$ CERNET Center, Tsinghua University, Beijing, 100084, China \\ aIshuo72@gmail.com; 'buoliang.taurus@gmail.com; 'guoliang.taurus@gmail.com; \\ dxing@cernet.edu.cn
}

Keywords: IPv4/IPv6 transition, network architecture, next-generation Internet.

\begin{abstract}
While IPv4 and IPv6 coexists in the current Internet, there are multiple transition mechanisms that provide IPv4 connectivity to or across IPv6 networks, and these mechanisms each usually requires its own specific Customer Premises Equipment (CPE) to function. In this article, we propose a specification whereby a single unified CPE can support multiple mainstream transition mechanisms, including Mapping of Address and Port (MAP-T, MAP-E), 464XLAT, Dual-Stack Lite (DS-Lite), and dual-stack. The unified CPE detects network configurations, and automatically adapts its functionalities.
\end{abstract}

\section{Introduction}

The exhaustion of IPv4 address space has become a critical problem the Internet is facing today [1]. IPv6 [2] was designed to solve this problem with a much larger address space, it also has many other technical advantages over IPv4. The deployment of IPv6 will significantly benefit the next-generation Internet.

While IPv4 and IPv6 coexists, many transition mechanisms are proposed to provide IPv4 connectivity to or across IPv6 networks. For many of such mechanisms, Customer Premises Equipment (CPE) is used for providing Internet access in homes and small offices. Since there are various kinds of such mechanisms, each requires its own specific CPE, a network administrator would typically have to choose the right type of CPE and configure them with corresponding parameters according to which transition mechanism is used.

In this article, we propose a specification whereby a single unified CPE can work with several mainstream transition mechanisms. Network administrator decides which mechanism to use, and configure the network accordingly, the unified CPE can automatically detect network configuration using DHCPv6 [3] for IPv6, and DHCPv4/IPCPv4 for IPv4, and then adjust itself to work with the mechanism.

There are other efforts trying to unify different transition mechanisms. [4] proposes a unified CPE supporting Softwire tunnelling mechanisms, while it is similar to our approach, our unified CPE supports a wider variety of mechanisms, including translation mechanisms and dual-stack. Also, compared to [4], this article is more specific, and provides implementation and experiments. [5] proposes a generic approach to unify IPv4/IPv6 transition using software defined network (SDN). This approach has great potential, but SDN centralizes management and control, making the network vulnerable to attacks and failures. Our approach leverages distributed network architecture, therefore provides more reliability.

The rest of this article is organized as follows: In section 2, we introduce the fundamental of IPv4/IPv6 transition mechanisms, and present the mechanisms that the unified CPE supports. In section 3, we discuss the behaviour of the unified CPE, including its working modes and how it 
works with DHCPv6. In section 4, we present implementation and experiments. In section 5, we conclude the article and address future work.

\section{IPv4/IPv6 transition mechanisms}

IPv6 is designed as a conservative extension of IPv4, but the structures of IPv4 and IPv6 are significantly different and incompatible, coexistence and transition problems emerge and hold up the IPv6 deployment. Many IPv4/IPv6 transition mechanisms are proposed to solve these problem, these mechanisms fall into 3 categories: dual-stack, tunnelling and translation [6], [7], as shown in Table 1.

Table 1: Transition Mechanisms

\begin{tabular}{l|l}
\hline Type & Mechanisms \\
\hline Dual-stack & N/A \\
Tunnelling & Lightweight 4over6, DS-Lite, MAP-E, ... \\
Translation & NAT64, 464XLAT, MAP-T, ... \\
\hline
\end{tabular}

Table 2: Comparison of Supported IP Transition Mechanisms

\begin{tabular}{l|llll}
\hline Mechanism & MAP-T & MAP-E & 464XLAT & DS-Lite \\
\hline Type & Translation & Tunnelling & Translation & Tunnelling \\
\hline Stateful/stateless & Stateless & Stateless & Stateful & Stateful \\
\hline $\begin{array}{l}\text { Coupling of IPv4/IPv6 } \\
\text { addresses }\end{array}$ & Yes & Yes & No & No \\
\hline $\begin{array}{l}\text { Support bidirectional } \\
\text { access? }\end{array}$ & Yes & Yes & No & No \\
\hline
\end{tabular}

Dual-stack is a trivial solution that requires hosts and infrastructure be upgraded to support IPv4 and IPv6. The unified CPE supports dual-stack, and also single-stack (IPv4-only/IPv6-only) as a fall-back mode.

Tunnelling mechanisms encapsulate packet of one address family inside packet of another. Tunnelling includes two types: the IPv6 over IPv4 tunnels are mainly used to connect IPv6 islands across IPv4 Internet during the early phase of the transition, while the IPv4 over IPv6 tunnels aim to provide IPv4 connectivity across newly deployed IPv6 networks. Our unified CPE supports IPv4 over IPv6 tunnels, MAP-E and Dual-Stack Lite (DS-Lite) in particular.

Translation mechanisms translate between packet headers in IPv6 and IPv4. Translation connects IPv6 network and IPv4 network, double translation provides IPv4 connectivity across IPv6 network, similar to tunnelling. Translation allows IPv4 to gradually phase out while IPv6 grows.

Our unified CPE supports mainstream transition mechanisms, including: MAP-T [8], MAP-E [9], 464XLAT [10], DS-Lite [11], and dual-stack. Table 2 shows a comparison of these mechanisms. 


\section{Unified CPE behavior}

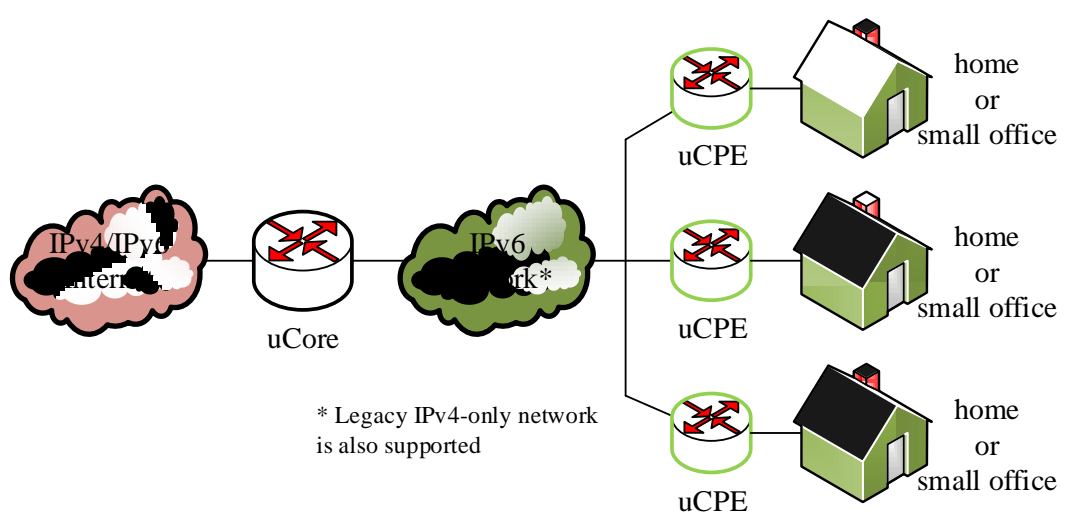

Fig.1: Network Topology for Unified CPE

Table 3: Unified CPE Function

\begin{tabular}{l|ll}
\hline Mechanism & uCore & uCPE \\
\hline MAP-T/E & BR & CE \\
464XLAT & PLAT & CLAT \\
DS-Lite & AFTR & B4 \\
Dual-stack & N/A & Router \\
\hline
\end{tabular}

Unified CPE working modes and mode decision Logic. The figure above shows the network topology in which unified CPE functions. Generally, unified CPE do client side encapsulation or translation for tunnelling and translation mechanisms, the unified CPE automatically adapts itself, as shown in Table 3.

The unified CPE can function in the following modes, ordered by preference. The preference order is:

Mode 1: MAP-T. MAP-T, as a stateless translation mechanism, provides good scalability, manageability, and routing efficiency. It is the preferable mechanism to push forward IPv4/IPv6 transition progress. So MAP-T working mode has the highest preference.

Mode 2: MAP-E. As a tunnelling mechanism, MAP-E maintains full IPv4 packet header during transmission, therefore provides more IPv4 transparency than translation mechanisms, but tunnelling mechanisms cannot support IPv4/IPv6 interconnection, therefore cannot promote IPv4/IPv6 transition.

Mode 3: 464XLAT. 464XLAT is a relatively simple translation mechanism, it requires minimal configuration on the CPE side. But as a stateful mechanism, it requires the provider-side translator (PLAT) to maintain session states, and it does not support bidirectional access.

Mode 4: DS-Lite. DS-Lite is a stateful tunnelling mechanism. It is relatively easy to deploy, but has many drawbacks, such as core statefulness, unable to support IPv4/IPv6 interconnection, unable to support bidirectional access, etc.

Mode 5: Dual-stack routing. This mode is a fall-back mode, activates when none of the above modes is applicable. In this mode, the CPE works like a normal IPv4/IPv6 home gateway router. For IPv4, it gets address via DHCPv4 or IPCPv4 (when PPPoE is enabled) from uplink, then 
assigns private IPv4 addresses to downlink via DHCPv4 and enables network address translation (NAT). For IPv6, it gets address and prefix via DHCPv6 from uplink, then delegates its dedicated IPv6 prefix by assigning addresses to downlink using DHCPv6 or SLAAC. IPv4 and IPv6 are totally decoupled in this mode, so the legacy IPv4-only network is also supported.

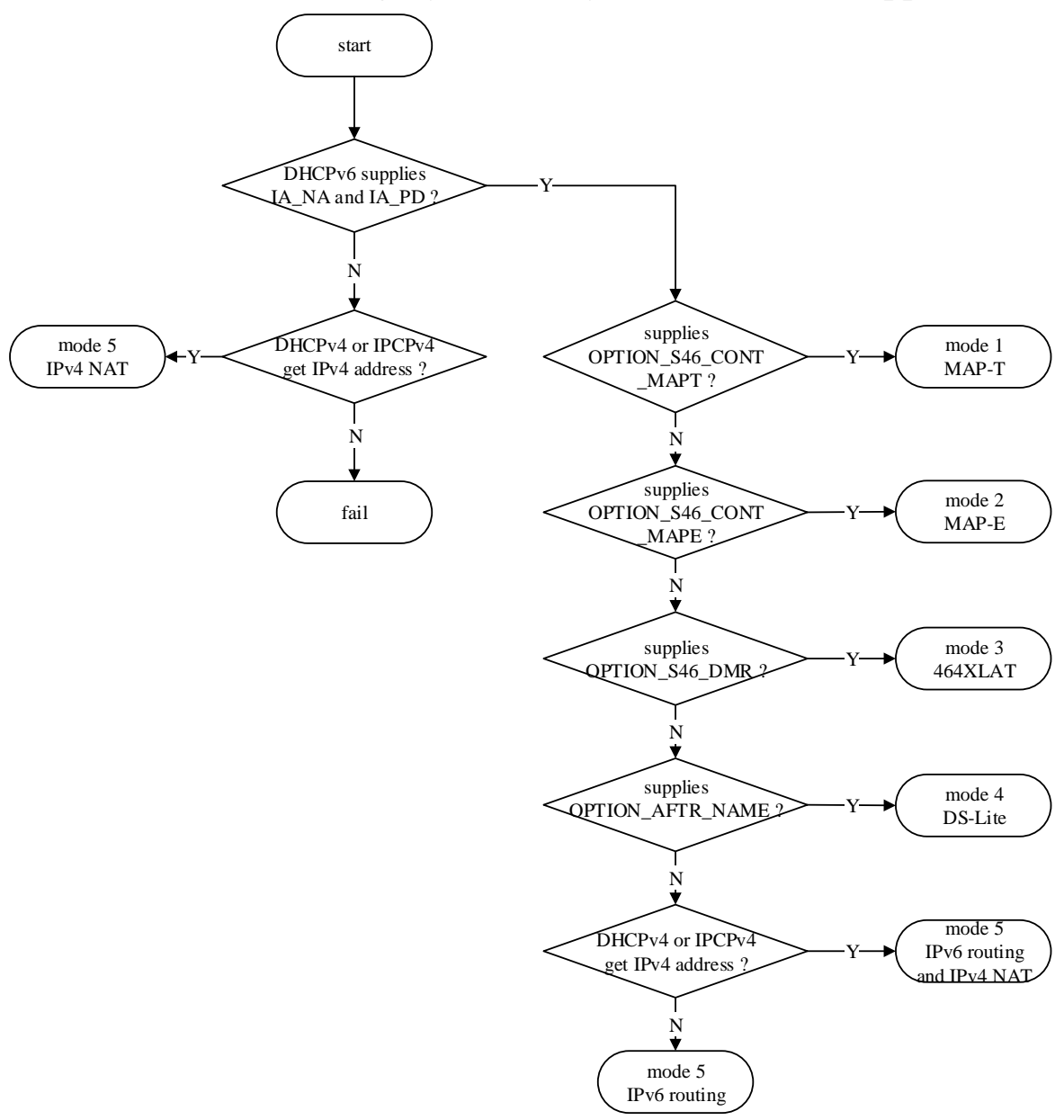

Fig.2: Unified CPE Mode Decision Logic Flow Chart

When more than one mode is available in the network side, the unified CPE will choose its working mode according to the above preference order. More specifically, when the unified CPE is enabled, it first sends a DHCPv6 request with all the 6 options above embedded, the provider network would reply with a subset of these 6 options according to which transition mechanisms are used, the CPE then decide which working mode to enable based on the received options and the preference order, if none of the supported mechanisms is detected, the unified CPE will fall back to dual-stack routing mode, in which it tries to get IPv4 configuration from uplink via DHCPv4/IPCPv4. Fig.2 shows the working mode decision logic, the specification of DHCPv6 options shown in the figure will be illustrated in section 3.2.

DHCPv6 Options Specification. DHCPv6 is the IPv6 version of DHCP, it can configure hosts with IPv6 addresses and IPv6 prefixes, it can also provide other configuration data using DHCPv6 options. DHCPv6 is used for the unified CPE to detect which transition mechanisms the provider network is configured with, and the corresponding configuration parameters.

[12] specifies DHCPv6 options for MAP-T/MAP-E. [13] specifies the AFTR-Name DHCPv6 option for DS-Lite. For 464XLAT, we borrow OPTION_S46_DMR defined in [12] to provision PLAT IPv6 prefix. Table 4 shows the DHCPv6 options needed for each transition mechanism. 
Table 4: DHCPv6 Options for Supported Transition Mechanisms

\begin{tabular}{l|llll}
\hline DHCPv6 option & MAP-T & MAP-E & 464XLAT & DS-Lite \\
\hline IA_NA & Y & Y & Y & Y \\
IA_PD & Y & Y & Y & Y \\
OPTION_S46_CONT_MAPT & Y & & & \\
OPTION_S46_CONT_MAPE & & Y & & \\
OPTION_S46_DMR & & & Y & \\
OPTION_AFTR_NAME & & & & Y \\
\hline
\end{tabular}

\section{Implementation and Experiments}

OpenWrt [14] is an extensible GNU/Linux distribution for embedded wireless routers. We built the unified CPE experimental implementation based on OpenWrt Barrier Breaker 14.07, installed packages include: ip, dnsmasq, ppp, ppp-mod-pppoe, radvd, odhcp6c, odhcpd, luci, etc.

We use odhcp6c as the DHCPv6 client, and wrote an odhcp6c user script to handle received DHCPv6 options and implement the logic shown in Fig.2. Some options are parsed by a modified version of mdpc [15]. We use a modified version of [16] for MAP-T/MAP-E CE and 464XLAT CLAT, and we use the ipip6 tunnel mode of ip program for DS-Lite B4.

We also implemented a LuCI [17] web page allowing users to toggle unified CPE functions, shown in Fig.3.

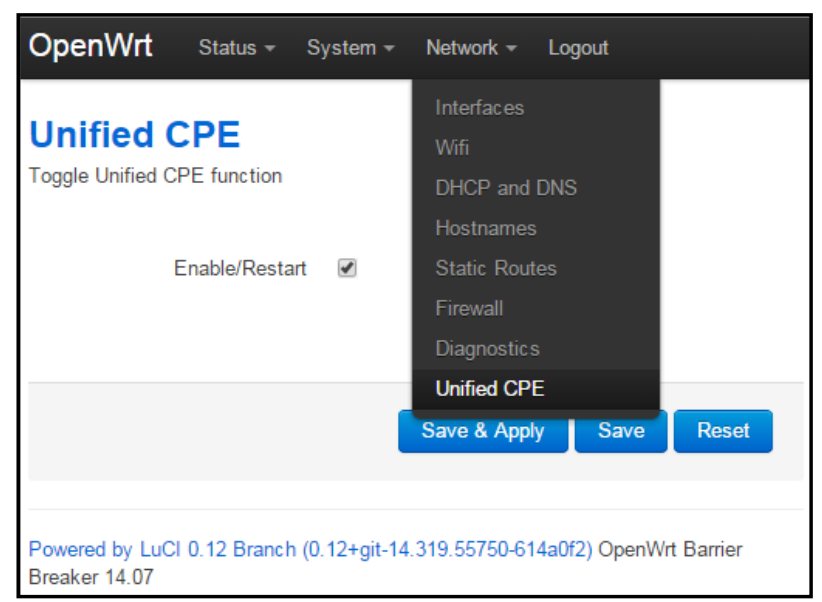

Fig.3: Unified CPE LuCI Web Page

We conduct simple in-lab experiments to demonstrate functionalities of the unified CPE. The network topology is shown in Fig.4. The uCPE is a TP-Link TL-WR703N router running OpenWrt with unified CPE functions implemented, all other boxes are hosts or virtual machines running Linux. uCore runs core translation/encapsulation for each mechanism. ISC DHCP server is used as DHCPv6/DHCPv4 Server, and is configured with parameters and options for the mechanisms.

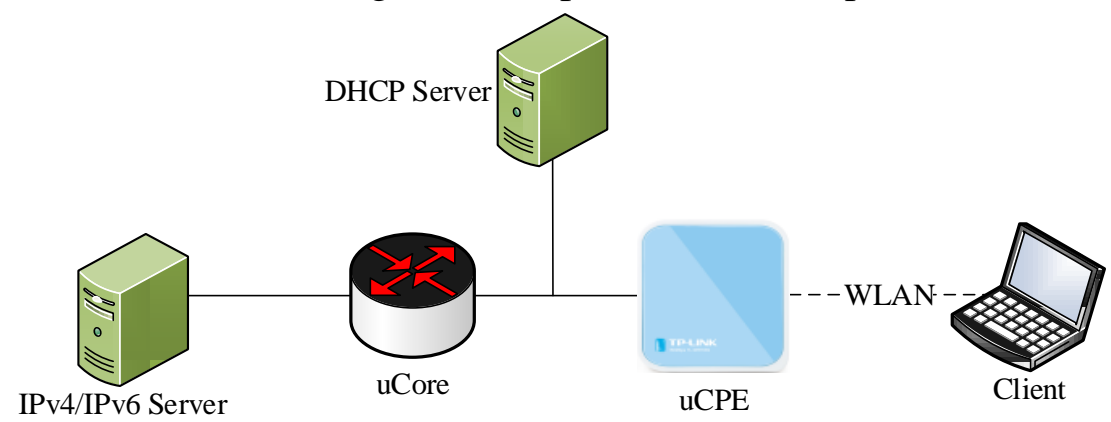

Fig.4: In-Lab Experiments Topology 


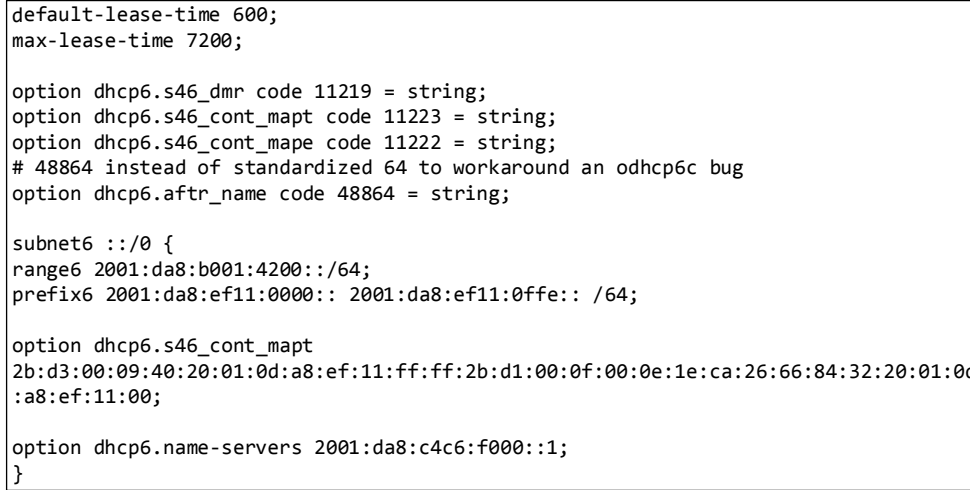

Fig.5: ISC DHCP Server DHCPv6 Configuration for MAP-T Mode

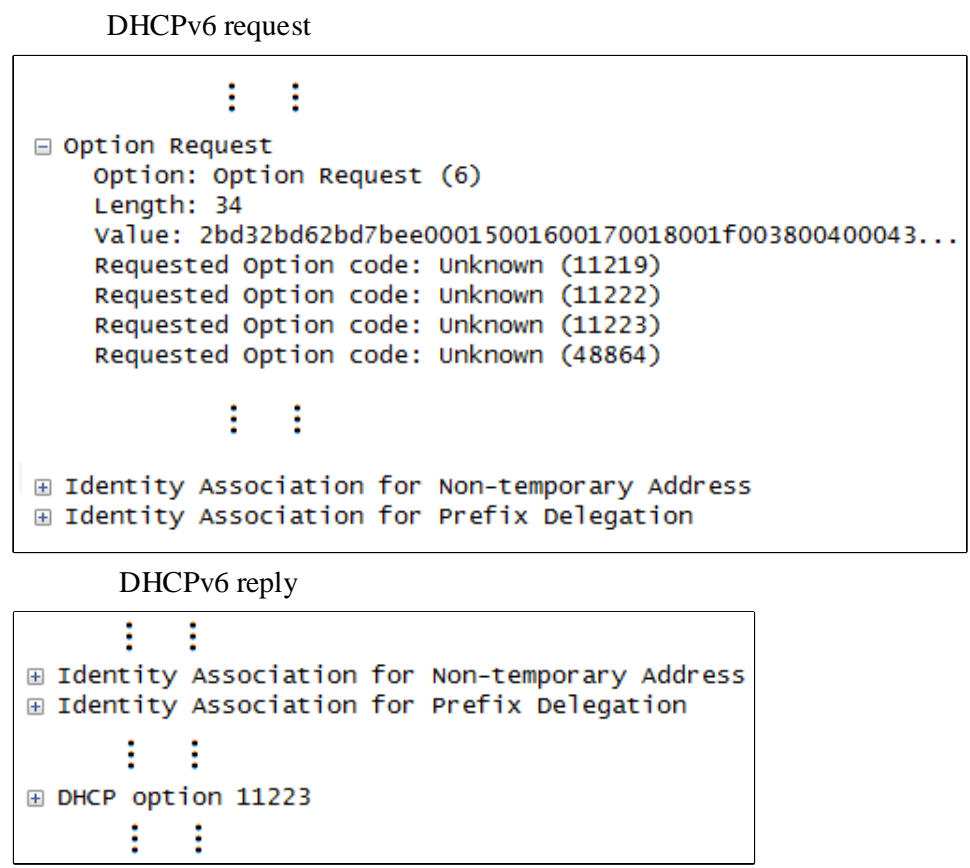

Fig.6: DHCPv6 Communication between CPE and DHCPv6 Server

To verify unified CPE functionality, we setup each mechanism, enable unified CPE, and then verify that the client can access the dual-stack server (ping, SSH, HTTP, etc.) using both IPv4 and IPv6. For example, if uCore is configured with MAP-T, we can serve DHCPv6 options indicating MAP-T mode by modifying DHCPv6 server configuration file as Fig.5 shows.

The CPE sends DHCPv6 request packet requesting all supported options, the DHCPv6 server then sends reply packet with IA_NA, IA_PD, and OPTION_S46_CONT_MAPT, as Fig.6 shows.

The CPE then automatically configures itself to work in MAP-T mode, we can verify the CPE working mode by checking whether the software module (ivictl) is running. The client can now access the dual-stack server with IPv4 and IPv6 through the CPE.

\section{Conclusions}

In this article, we proposed a specification whereby a unified CPE can support mainstream IPv4/IPv6 transition mechanisms including MAP-T, MAP-E, 464XLAT, DS-Lite, and dual-stack. The unified CPE can detect the uplink network configuration, and automatically choose the suitable 
working mode. Compared to traditional CPE, the unified CPE saves the customers from choosing specific CPE, and supports automatic and unattended operation, therefore is much more convenient and cost-effective. It can greatly improve the flexibility of IPv6 deployment, as a result, push forward the IPv4-to-IPv6 transition process.

As IPv6 grows and transition mechanisms evolve, the specifications of them might change, and there might appear new mechanisms. In such cases, the idea of the unified CPE will still be viable, but the specifications will need to be modified and extended to reflect the changes and prevent ambiguity.

\section{References}

[1] Huston G., IPv4 Address Report, http://www.potaroo.net/tools/ipv4/

[2] Deering S \& Hinden R, RFC 2460: Internet Protocol, 1998.

[3] Droms R., Droms R., Bound J., Volz B., Lemon T., Perkins C., \& Carney M., RFC 3315: Dynamic host configuration protocol for IPv6 (DHCPv6), 2003.

[4] Boucadair M. Farrer I. Perreault S. \& Sivakumar S., Unified IPv4-in-IPv6 Softwire CPE, draft-ietf-softwire-unified-cpe-01 (work in progress), 2013.

[5] Xia W., Tsou T., Lopez D. R., Sun Q., Lu F. \& Xie H, A software defined approach to unified IPv6 transition, SIGCOMM, pp. 547-548, 2013.

[6] Baker F., Li X., Bao C. \& Yin K, Framework for IPv4/IPv6 Translation, RFC 6144, 2011.

[7] Wang X. F., Wu J. P. \& Cui Y., Survey of Internet IPv6 Transition Technologies, Journal of Chinese Computer Systems, 27(3), 2006.

[8] Li X., Bao C., Dec W., Troan O., Matsushima S. \& Murakami T. Mapping of Address and Port using Translation (MAP-T), draft-ietf-softwire-map-t-08 (work in progress), 2014.

[9] Troan O., Dec W., Li X., Bao C., Matsushima S., Murakami T. \& Taylor T., Mapping of Address and Port with Encapsulation (MAP), draft-ietf-softwire-map-12 (work in progress), 2014.

[10]Mawatari M., Kawashima M. \& Byrne C., 464XLAT: Combination of stateful and stateless translation, RFC 6877, 2013.

[11]Durand A., Droms R., Woodyatt J. \& Lee Y., Dual-Stack Lite Broadband Deployments Following IPv4 Exhaustion, RFC 6333, 2011.

[12]Mrugalski T., Troan O., Farrer I., Perreault S., Dec W., Bao C., Yeh L. \& Deng X., DHCPv6 Options for configuration of Softwire Address and Port Mapped Clients, draft-ietf-softwire-map-dhcp-11 (work in progress), 2014.

[13]Hankins D. \& Mrugalski T., Dynamic Host Configuration Protocol for IPv6 (DHCPv6) Option for Dual-Stack Lite, RFC 6334, 2011.

[14]OpenWrt, https://openwrt.org/

[15]GitHub, Inc., ayourtch/mdpc, https://github.com/ayourtch/mdpc

[16] GitHub, Inc., cernet/MAP, https://github.com/cernet/MAP

[17] LuCI - Technical Reference. http://wiki.openwrt.org/doc/techref/luci 